\title{
Investigation of the role of tumor necrosis factor-like weak inducer of apoptosis in non-small cell lung cancer
}

\author{
WEI-AN CHANG ${ }^{1,2 *}$, MENG-CHI YEN ${ }^{3 *}$, JEN-YU HUNG $^{2,4}$, CHIH-JEN YANG ${ }^{2,4}$, SHU-FANG JIAN ${ }^{1}$, \\ I-JENG YEH ${ }^{1,3,4}$, KUAN-TING LIU ${ }^{1,3,4}$, YA-LING HSU ${ }^{5}$ and PO-LIN KUO ${ }^{1,5}$ \\ ${ }^{1}$ Graduate Institute of Clinical Medicine, College of Medicine, Kaohsiung Medical University; \\ ${ }^{2}$ Division of Pulmonary and Critical Care Medicine, and ${ }^{3}$ Department of Emergency Medicine, \\ Kaohsiung Medical University Hospital; ${ }^{4}$ School of Medicine and ${ }^{5}$ Graduate Institute of Medicine, \\ College of Medicine, Kaohsiung Medical University, Kaohsiung 807, Taiwan, R.O.C.
}

Received July 1, 2017; Accepted December 1, 2017

DOI: 10.3892/or.2017.6141

\begin{abstract}
Several of the soluble inflammatory molecules such as cytokines and chemokines are involved in the regulation of cancer behaviors. Tumor necrosis factor (TNF)-like weak inducer of apoptosis (TWEAK) is a member of the TNF superfamily and is a ligand of fibroblast growth factor inducible 14 (Fn14). TWEAK/Fn14 signaling pathways promote tumor progression in several types of human cancer. In the present study, we investigated the role of TWEAK through bioinformatic assay, in vitro experiments, and serum levels in patients with non-small cell lung cancer (NSCLC). Our results indicated that TWEAK expression in normal tissues was higher than that in lung cancer tissues. In contrast, relatively higher Fn14 expression was detected in lung cancer tissues compared to normal tissues. Recombinant TWEAK treatment did not enhance and inhibit the proliferation and migration of human NSCLC cell lines including A549, H1299, CL1-0 and CL1-5. In addition, the serum concentration of TWEAK in normal controls was significantly higher than that in NSCLC patients. However, the TWEAK levels did not show significant difference in regards to TNM stage, cell type and metastasis status in the sera of NSCLC patients. In summary, the present study suggests that a low serum level of TWEAK may be a feature of NSCLC, and the role of TWEAK-mediated pathways warrant further investigation.
\end{abstract}

Correspondence to: Professor Ya-Ling $\mathrm{Hsu}$ or Professor Po-Lin Kuo, Graduate Institute of Medicine, College of Medicine, Kaohsiung Medical University, 100 Shih-Chuan 1st Road, Kaohsiung 807, Taiwan, R.O.C.

E-mail: hsuyl326@gmail.com

E-mail: kuopolin@seed.net.tw

${ }^{*}$ Contributed equally

Key words: tumor necrosis factor-like weak inducer of apoptosis, fibroblast growth factor inducible 14, non-small cell lung cancer

\section{Introduction}

Lung cancer is one of the most lethal malignancies worldwide (1). Targeted therapy drugs that target receptor tyrosine kinases including epidermal growth factor receptor, HER 2/neu, echinoderm microtubule-associated proteinlike 4 (EML4) and anaplastic lymphoma kinase (ALK) improve patient outcomes (2-4). In addition, immunotherapy agents such as cytotoxic T-lymphocyte-associated antigen 4 (CTLA-4), programmed death 1 (PD-1) receptor and its ligands (PD-L1) have been approved for the treatment of lung cancer (5). However, some lung cancer patients exhibit no or limited responses to these treatments. Therefore, identifying unknown tumor-associated molecules and signaling pathways is still a critical strategy by which to develop novel therapeutic strategies.

Recent studies indicate that soluble factors are key factors that affect the behaviors of tumor cells in the tumor microenvironment. Extracellular matrix component-induced signaling pathways regulate the migration and proliferation of tumor cells (6). Various soluble factors including vascular endothelial growth factor A, platelet derived growth factor and osteopntin are involved in the malignant development of lung cancer (7). In addition, tumor-infiltrating macrophage and myeloid-derived suppressor cells are also important components in the tumor microenvironment $(8,9)$. These immune cells that secrete cytokines and chemokines are important factors in malignancy. Chemokine (C-X-C motif) ligand 5 (CXCL5), CXCL8, CXCL12, C-C motif chemokine ligand 5 (CCL5), and receptors $\mathrm{C}-\mathrm{X}-\mathrm{C}$ motif chemokine receptor 4 (CXCR4) and CXCR8 play a role in lung cancer progression (10-12). Tumor necrosis factor- $\alpha$ (TNF- $\alpha$ ) is one of the most important inflammatory mediators and induces the apoptosis of tumor cells (13). In contrast, TNF- $\alpha$ also mediates angiogenesis and chronic inflammation through the Jun kinase/nuclear factor- $\mathrm{\kappa B}$ (NF- $\mathrm{\kappa B}$ ) signaling pathway (14). This evidence implies that investigation of soluble factor-associated signaling pathways may identify novel tumor markers or therapeutic targets.

Tumor necrosis factor (TNF)-like weak inducer of apoptosis (TWEAK)/tumor necrosis factor superfamily member 12 
(TNFSF12) is a member of the TNF superfamily and a ligand of fibroblast growth factor inducible 14 (Fn14)/TNF receptor superfamily member 12A (TNFRSF12A) (15). TWEAK activates the $\mathrm{NF}-\kappa \mathrm{B}$ signaling pathway through autocrine and paracrine manners (15). Overexpression of Fn14 is correlated with poor treatment outcome in prostate cancer and is a negative prognostic factor in breast and gastric cancer and glioma (16-19). In addition, TWEAK promotes the invasive capacity in prostate and glioma cells via activation of the $\mathrm{NF}-\kappa \mathrm{B}$ signaling pathway $(20,21)$. Blockage of TWEAK may be a strategy by which to inhibit the metastasis of breast cancer and glioma (22). However, it is interesting to note that TWEAK overexpression in melanoma inhibits its cell growth and invasion even though the $N F-\kappa B$ signaling pathway is activated (21). Decreased TWEAK expression and increased Fn14 expression are observed in cervical cancer cell lines and clinical specimens of squamous cervical carcinoma (23). These findings suggest that the role of the TWEAK/Fn14 signaling pathway is diverse in different types of cancers.

In lung cancer, Src, hepatocyte growth factor receptor (HGFR/MET) and EGFR mutation (exon 19 deletion)-triggered signaling pathways contribute to Fn 14 expression (24-26). Src- and MET-driven invasion correlates with FN14 expression $(24,26)$. Although the expression of Fn14 is associated with tumor progression, the role of TWEAK is not fully understood in lung cancer. We investigated these issues by analysis of bioinformatic tools and in vitro human cancer cell lines. In addition, the TWEAK levels in serum samples of lung cancer patients and healthy donors were determined.

\section{Materials and methods}

Bioinformatic analysis. TWEAK and Fn14 expression in clinical lung cancer samples and non-tumor samples were determined by accessing the Oncomine Research Edition (http://www.oncomine.org, v4.5; Thermo Fisher Scientific, Inc., Waltham, MA, USA). The expression pattern of TWEAK and Fn14 in tumor and non-tumor regions was obtained from six cohorts including Bhattacharjee's (27), Hou's (28), Landi's (29), Selamat's (30) and Su's cohort (31). The TWEAK expression pattern in 151 lung cancer cell lines was obtained from the Cancer Cell Line Encyclopedia (CCLE) database (expression data was obtained from Oncomine) (32) and the heatmap was made by a web-based tool Morpheus (https://software.broadinstitute.org/morpheus/). TWEAK and Fn14 expression in Calu-3, A549, H1299, CL1-0, CL1-5 and $\mathrm{H} 209$ cells was obtained from GEO datasets (accession no. GSE7670 and GSE36133) (31,32).

Assessment of the patient survival rate on KM Plotter database. The survival analysis in lung cancer patients with different expression levels of TWEAK and Fn14 was performed through the KM-Plotter database (33). The prognostic value of each gene was analyzed by splitting patient samples into two groups by the median, after the subtype (adenocarcinoma and squamous cell carcinoma) of lung cancer was chosen. The relapse-free survival rate was analyzed (2016.04.08 update, the lung cancer database included 1,926 samples).
Cell culture. The human lung adenocarcinoma cell lines, CL1-0 and CL1-5, were kindly provided by Dr Pan-Chyr Yang (Department of Internal Medicine, National Taiwan University Hospital, Taipei, Taiwan) (34). Human lung carcinoma cell line A549 and a non-small cell lung cancer cell line H1299 were purchased from the American Type Culture Collection (ATCC; Manassas, VA, USA). CL1-0, CL1-5, A549 and H1299 cells were maintained in RPMI-1640 medium which was supplemented with $10 \%$ fetal bovine serum (FBS) and $1 \%$ penicillin-streptomycin (Life Technologies, Grand Island, NY, USA) in an incubator at $37^{\circ} \mathrm{C}$ in $5 \% \mathrm{CO}_{2}$.

Recombinant protein and chemicals. Recombinant human TWEAK was obtained from R\&D Systems (Minneapolis, MN, USA). All chemicals and buffer were purchased from Sigma-Aldrich (St. Louis, MO, USA).

Proliferation assay (WST-1 assay). The effect of TWEAK protein on cell proliferation was evaluated by WST-1 (4-[3-(4-iodophenyl)-2-(4-nitrophenyl)-2H-5-tetrazolio]-1,3benzene disulfonate) (Clontech, Mountain View, CA, USA). Briefly, 5x10³ CL1-0, CL1-5, A549 and H1299 cells were respectively seeded in 96 -well plates overnight. Before TWEAK treatment, the medium was replaced by RPMI- 1640 with $0.5 \%$ FBS for $6 \mathrm{~h}$. The proliferation rate was determined at a wavelength of $450 \mathrm{~nm}$ on a microplate spectrophotometer (PowerWave X340; BioTek, Winooski, VT, USA).

Transwell migration assay. Transwell cell migration assay was performed through QCM ${ }^{\mathrm{TM}}$ 24-well Cell Migration Assay and Invasion System uncoated $8-\mu \mathrm{m}$ pore size polycarbonate membranes (Millipore, St. Charles, MO, USA) according to the manufacturer's instructions. A549, CL1-0, CL1-5 or H1299 $\left(3 \times 10^{5}\right)$ cells were seeded into a 24 -well insert in $300 \mu \mathrm{l}$ of serum-free medium, while $500 \mu 1$ medium with $10 \%$ FBS was placed in the lower chamber and then were incubated for $24 \mathrm{~h}$. The Transwell membrane was fixed with $4 \%$ formaldehyde solution followed by $1 \%$ crystal violet staining. After removal of the cells on the upper surface via a cotton swab, images of the bottom membrane were then captured using an Olympus inverted microscope at x100 magnification.

Wound healing assay. Lung cancer cells $\left(2 \times 10^{5}\right)$ were seeded into 24-well plates. When cells reached a complete confluent monolayer, a scratch was made using a $200-\mu 1$ pipette tip. Cell debris was removed by phosphate-buffered saline washing after scratching. Then, the cells were incubated in RMPI-1640 medium with $10 \%$ FBS for $24 \mathrm{~h}$. The images and quantitative results were performed via Leica Applications Suite version 4.5.0 ${ }^{\mathrm{TM}}$ (LAS v4.5) software (Leica Microsystems, Wetzlar, Switzerland).

Quantification of TWEAK levels in serum. The use of the serum samples in the present study was approved by the Institutional Review Board of Kaohsiung Medical University Hospital. Serum samples from the patients with lung cancer and healthy donors were obtained at Kaohsiung Medical University Hospital (Kaohsiung, Taiwan) after patients provided informed consent. Blood from all donors was drawn 

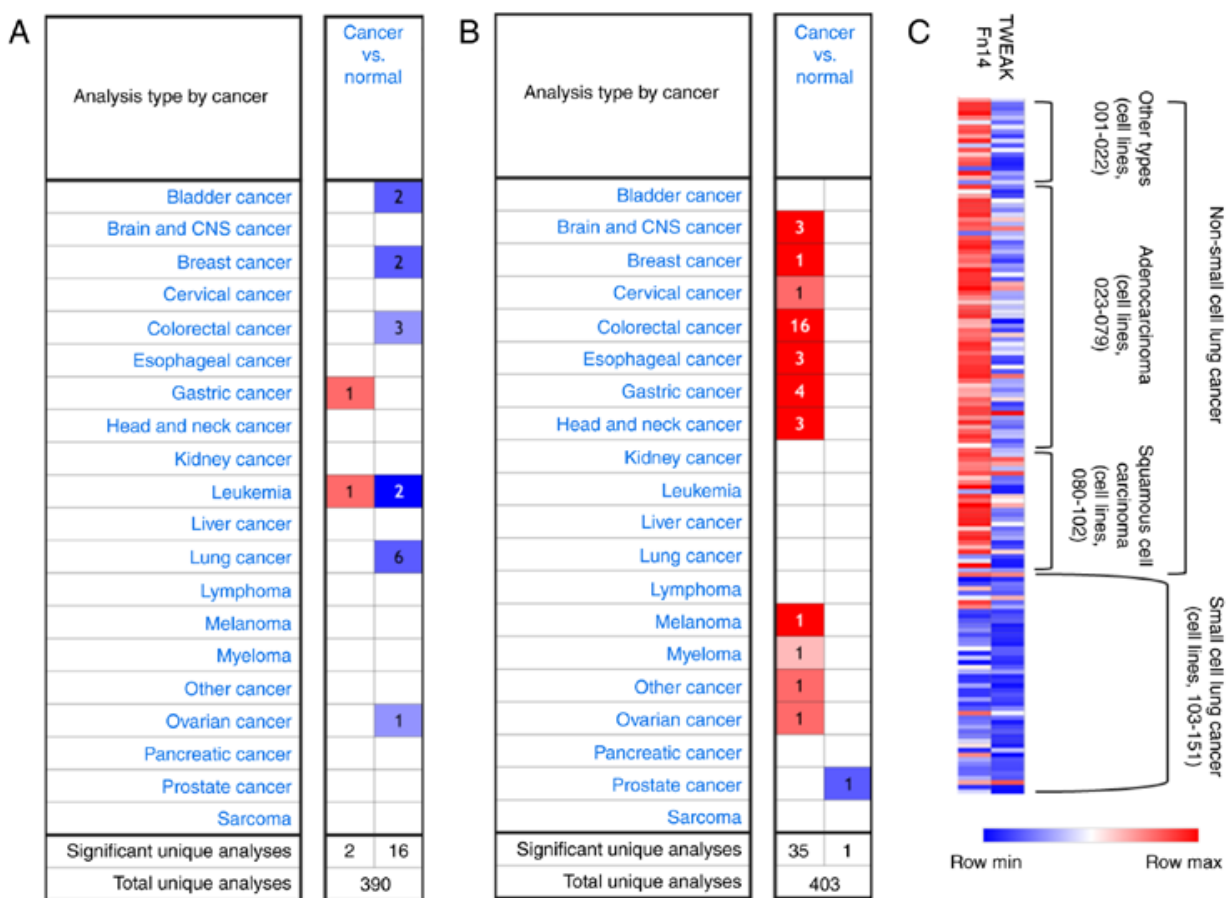

Figure 1. TWEAK and Fn14 mRNA levels in various human cancer types and lung cancer cell lines. mRNA levels of (A) TWEAK and (B) Fn14 in various cancer types are shown. The data were obtained from Oncomine database in a threshold: P-value of 1E-4, fold-change of 2, and gene ranking of $10 \%$. Red and blue color in each column respectively indicates overexpression and under-expression of TWEAK and Fn14 in human cancer. (C) Heatmap displaying mRNA expression of TWEAK and Fn14 in different types of 151 lung cancer cell lines through CCLE database. Detailed information of each cell is shown in Table I according to the number of the cell.
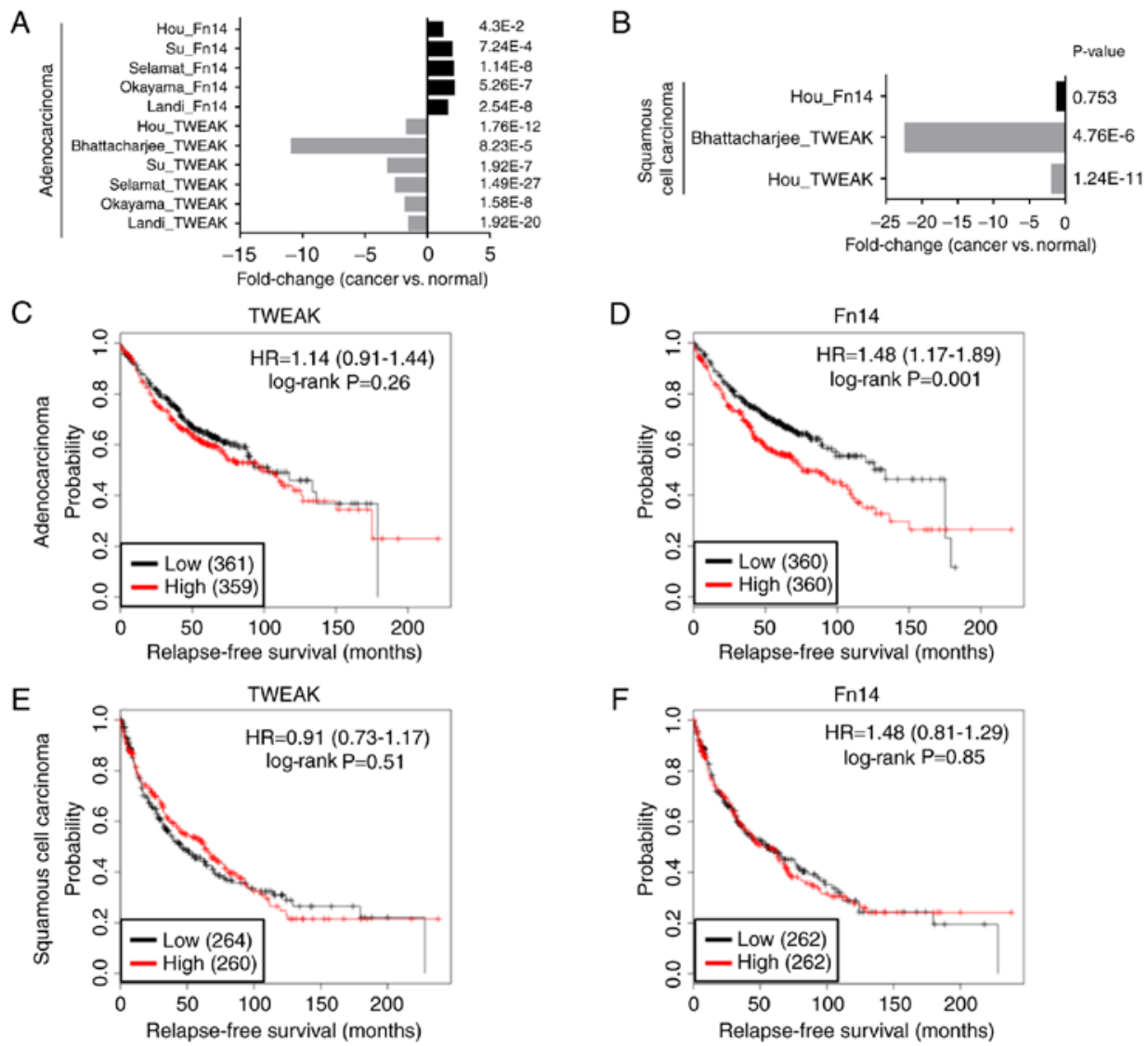

Figure 2. The role of TWEAK and Fn14 in lung adenocarcinoma and squamous cell carcinoma. The fold-change of TWEAK and Fn14 in (A) lung adenocarcinoma and (B) squamous cell carcinoma is shown. The results were collected from six independent cohorts (including Hou, Su, Selamat, Okayama, Landi, and Bhattacharjee cohorts on Oncomine database) which include TWEAK or Fn14 expression. The survival curve was analyzed by the KM plotter database. The survival curve comparing the patients with high (red) and low (black) is shown. The survival curve of (C) TWEAK and (D) Fn14 in adenocarcinoma, and (E) TWEAK and (F) Fn14 in squamous cell carcinoma. 
Table I. A complete list of cell lines in each group.

Non-small cell lung cancer

\begin{tabular}{|c|c|c|c|}
\hline $\begin{array}{l}\text { Other types } \\
(001-022)\end{array}$ & $\begin{array}{l}\text { Adenocarcinoma } \\
\quad(023-079)\end{array}$ & $\begin{array}{l}\text { Squamous cell } \\
\text { carcinoma } \\
(080-102)\end{array}$ & $\begin{array}{l}\text { Small cell lung cancer } \\
\qquad(103-151)\end{array}$ \\
\hline Calu-6 (001) & SK-LU-1 (023), NCI-H2023 (024) & NCI-H1703 (080) & NCI-H1048 (103), SBC-5 (104) \\
\hline COR-L23 (002) & NCI-H1693 (025), Calu-3 (026) & NCI-H1869 (081) & NCI-H1092 (105), SHP-77 (106) \\
\hline IA-LM (003) & NCI-H1793 (027), COR-L105 (028) & SK-MES-1 (082) & NCI-H1341 (107), NCI-H211 (108) \\
\hline LCLC-103H (004) & NCI-H2030 (029), COLO-699 (030) & LUDLU-1 (083) & DMS 114 (109), SW 1271 (110) \\
\hline NCI-H460 (005) & NCI-H23 (031), NCI-H1792 (032) & NCI-H2170 (084) & NCI-H1339 (111), NCI-H1694 (112) \\
\hline NCI-H1581 (006) & HCC-44 (033), NCI-H2009 (034) & EBC-1 (085) & NCI-H1184 (113), COLO 668 (114) \\
\hline NCI-H661 (007) & NCI-H1944 (035), NCI-H1651 (036) & NCI-H520 (086) & COR-L24 (115), COR-L311 (116) \\
\hline LCLC-97TM1 (008) & MOR/CPR (037), NCI-H2228 (038) & RERF-LC-AI (087) & COR-L47 (117), COR-L88 (118) \\
\hline NCI-H727 (009) & NCI-H1975 (039), NCI-H2085 (040) & HCC-15 (088) & COR-L95 (119), CPC-N (120) \\
\hline A549 (010) & NCI-H1355 (041), Hs 229.T (042) & LOU-NH91 (089) & DMS 153 (121), DMS 273 (122) \\
\hline BEN (011) & NCI-H1648 (043), NCI-H1573 (044) & NCI-H226 (090) & DMS 454 (123), DMS 53 (124) \\
\hline LU99 (012) & NCI-H522 (045), NCI-H1563 (046) & HARA (091) & DMS 79 (125), HCC-33 (126) \\
\hline LU65 (013) & NCI-H1373 (047), RERF-LC-MS (048) & Sq-1 (092) & NCI-H1105 (127), NCI-H1436 (128) \\
\hline NCI-H1915 (014) & NCI-H2122 (049), DV-90 (050) & Calu-1 (093) & NCI-H146 (129), NCI-H1618 (130) \\
\hline NCI-H1299 (015) & NCI-H2087 (051), NCI-H1568 (052) & LC-1/sq-SF (094) & NCI-H1836 (131), NCI-H1876 (132) \\
\hline NCI-H810 (016) & HCC-78 (053), PC-14 (054) & SW 900 (095) & NCI-H1930 (133), NCI-H196 (134) \\
\hline NCI-H2444 (017) & HCC4006 (055), HCC2935 (056) & KNS-62 (096) & NCI-H1963 (135), NCI-H2029 (136) \\
\hline NCI-H2172 (018) & HCC827 (057), ABC-1 (058) & EPLC-272H (097) & NCI-H2081 (137), NCI-H209 (138) \\
\hline NCI-H1155 (019) & HCC-1171 (059), HCC-2279 (060) & HCC-95 (098) & NCI-H2141 (139), NCI-H2171 (140) \\
\hline CAL-12T (020) & Hs 618.T (061), LXF-289 (062) & HLF-a (099) & NCI-H2196 (141), NCI-H2227 (142) \\
\hline NCI-H2106 (021) & NCI-H1395 (063), NCI-H1435 (064) & LK-2 (100) & NCI-H446 (143), NCI-H510 (144) \\
\hline \multirow[t]{9}{*}{ NCI-H2110 (022) } & NCI-H1437 (065), NCI-H1623 (066) & NCI-H1385 (101) & NCI-H524 (145), NCI-H526 (146) \\
\hline & NCI-H1734 (067), NCI-H1755 (068) & RERF-LC-Sq1 (102) & NCI-H69 (147), NCI-H82 (148) \\
\hline & NCI-H1838 (069), NCI-H2126 (070) & & NCI-H841 (149), NCI-H889 (150) \\
\hline & NCI-H2291 (071), NCI-H2342 (072) & & SCLC-21H (151) \\
\hline & NCI-H2347 (073), NCI-H2405 (074) & & \\
\hline & NCI-H838 (075), NCI-H854 (076) & & \\
\hline & RERF-LC-Ad1 (077) & & \\
\hline & RERF-LC-Ad2 (078) & & \\
\hline & RERF-LC-KJ (079) & & \\
\hline
\end{tabular}

Each cell line was matched with the number in parentheses which is shown in Fig. 1C.

in serum separation tubes and then stored in aliquots at $-80^{\circ} \mathrm{C}$. The serum levels of TWEAK were quantitated using MILLIPLEX MAP Human Cancer/Metastasis Biomarker Magnetic Bead Panel (Millipore) according to the manufacturer's instructions. Data were acquired on Luminex xMAP technology (Millipore). For concentration calculation, a cubic spline-fit method was performed using the Milliplex Analyst Software (Viagene Tech, Carlisle, MA, USA).

Statistical analysis. All bar graphs and statistics were performed using GraphPad Prism 7 (GraphPad Software, San Diego, CA, USA). Student's t-test and one-way ANOVA were respectively used for analysis of the difference between two groups and more than two groups. P-value $<0.05$ was considered to indicate a statistically significant difference.

\section{Results}

Evaluation of TWEAK and Fn14 expression in human lung cancer samples from online microarray databases. Previous studies have revealed that TWEAK and Fn14 are associated with the progression of several human cancers. In the present study, the expression patterns of TWEAK and Fn14 in human cancers were evaluated through an online database Oncomine (Fig. 1A and B). Compared to TWEAK, high expression of Fn14 was observed in multiple human cancer types except bladder, kidney, leukemia, liver and lung cancer. In Fig. 1A, six independent datasets showed that TWEAK expression in lung cancer samples was significantly lower than that in normal samples. Small cell lung cancer (SCLC; $\sim 10-15 \%$ ) and non-small cell lung cancer (NSCLC; 85-90\%) 
A

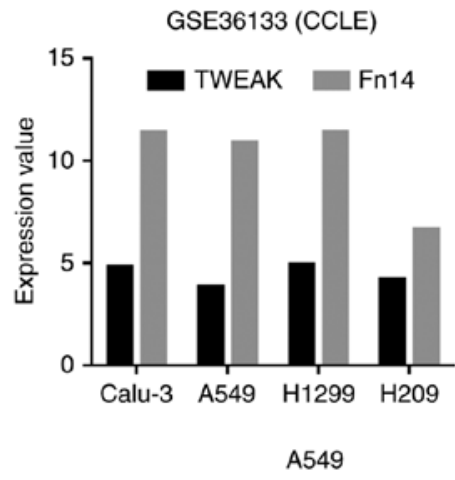

C

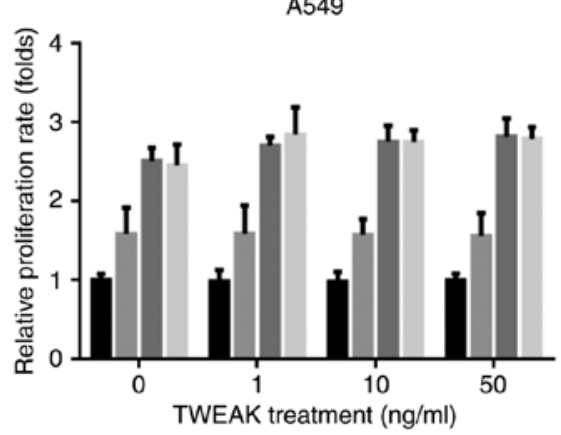

E

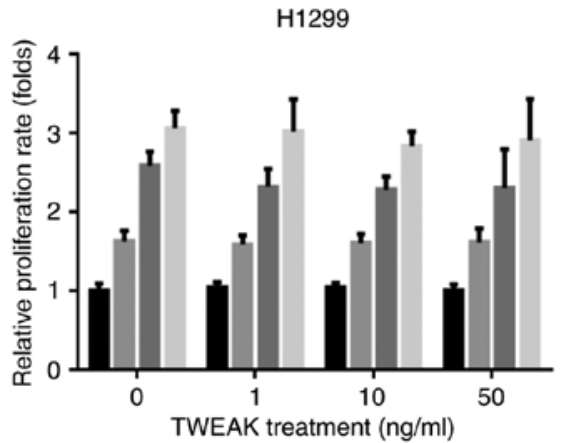

B

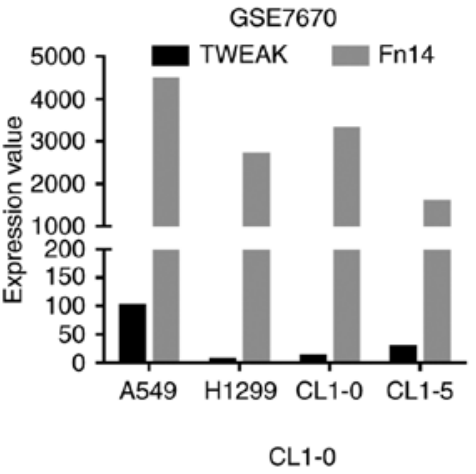

D

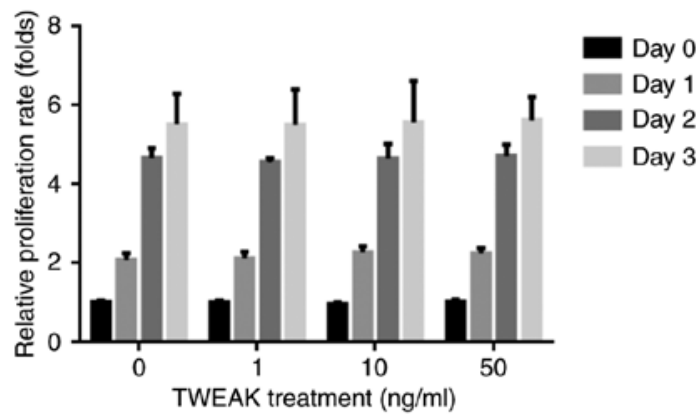

F

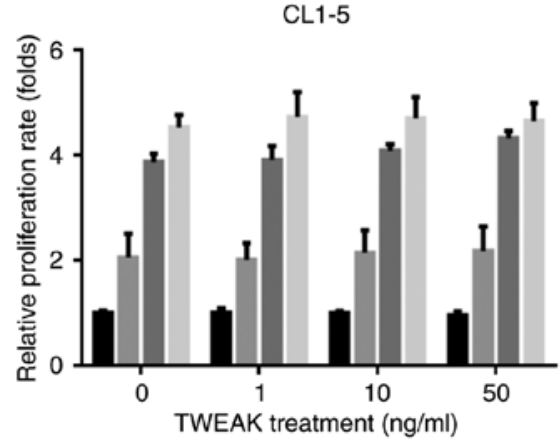

Figure 3. Effect of TWEAK treatment on cell growth. Expression of TWEAK and Fn14 expression through online datasets (A) GSE36133 and (B) GSE7670. Lung cancer cells were treated with recombinant TWEAK protein after $5 \times 10^{3}$ cells were plated and then incubated overnight. The cell growth of (C) A54 (D) CL1-0, (E) H1299 and (F) CL1-5 was evaluated by WST-1 assay from day 0-3 after TWEAK treatment. There is no significant difference among all groups in all cell lines.

are two major types of lung cancer and adenocarcinoma, squamous cell and large cell carcinomas, are main types of NSCLC (35). We further analyzed mRNA expression patterns in 151 human lung cancer samples from the Cancer Cell Line Encyclopedia database (GSE36133) (32). In general, Fn14 expression was found to be relatively low in SCLC compared to NSCLC (Fig. 1C, the list of complete cell lines is shown in Table I). Furthermore, most NSCLC cases expressed relatively low levels of TWEAK and high levels of Fn14. These results suggest that the role of the TWEAK/Fn14 signaling pathways may be different between NSCLC and SCLC.

Adenocarcinoma and squamous cell carcinoma are two major types of NSCLC. In order to further investigate the role of the TWEAK/Fn14 signaling pathways, six independent microarray datasets were collected on the Oncomine database and expression patterns in adenocarcinoma and squamous cell carcinoma were investigated. In adenocarcinoma, TWEAK expression was found to be lower and Fn14 expression was higher compared to the normal among all selected datasets (Fig. 2A). Since the number of squamous cell carcinoma patients was less than the number of adenocarcinoma patients in the clinic, the expression of TWEAK and Fn14 was only available in 1 and 2 datasets, respectively. Relatively low TWEAK expression was observed in squamous cell carcinoma in comparison with the normal cells (Fig. 2B). The correlation of TWEAK and Fn14 expression and the survival rate was evaluated by KM Plotter database. In Fig. 2C-F, high expression of Fn14 was found to be associated with poor outcome in adenocarcinoma. However, the survival rate was not associated with TWEAK in both adenocarcinoma and squamous cell carcinoma. This evidence through bioinformatic analysis suggests that low TWEAK expression may be a characteristic of NSCLC.

Effect of exogenous TWEAK treatment on different types of human NSCLC cell lines. To confirm the role of TWEAK and to mimic the effect of exogenous TWEAK in the tumor microenvironment, four human NSCLC cell lines including adenocarcinoma CL1-0 and CL1-5, lung carcinoma A549, and NSCLC H1299 were treated with recombinant TWEAK. The expression of Fn14 in these cells was firstly evaluated in the CCLE dataset (GSE36133) and another online dataset 

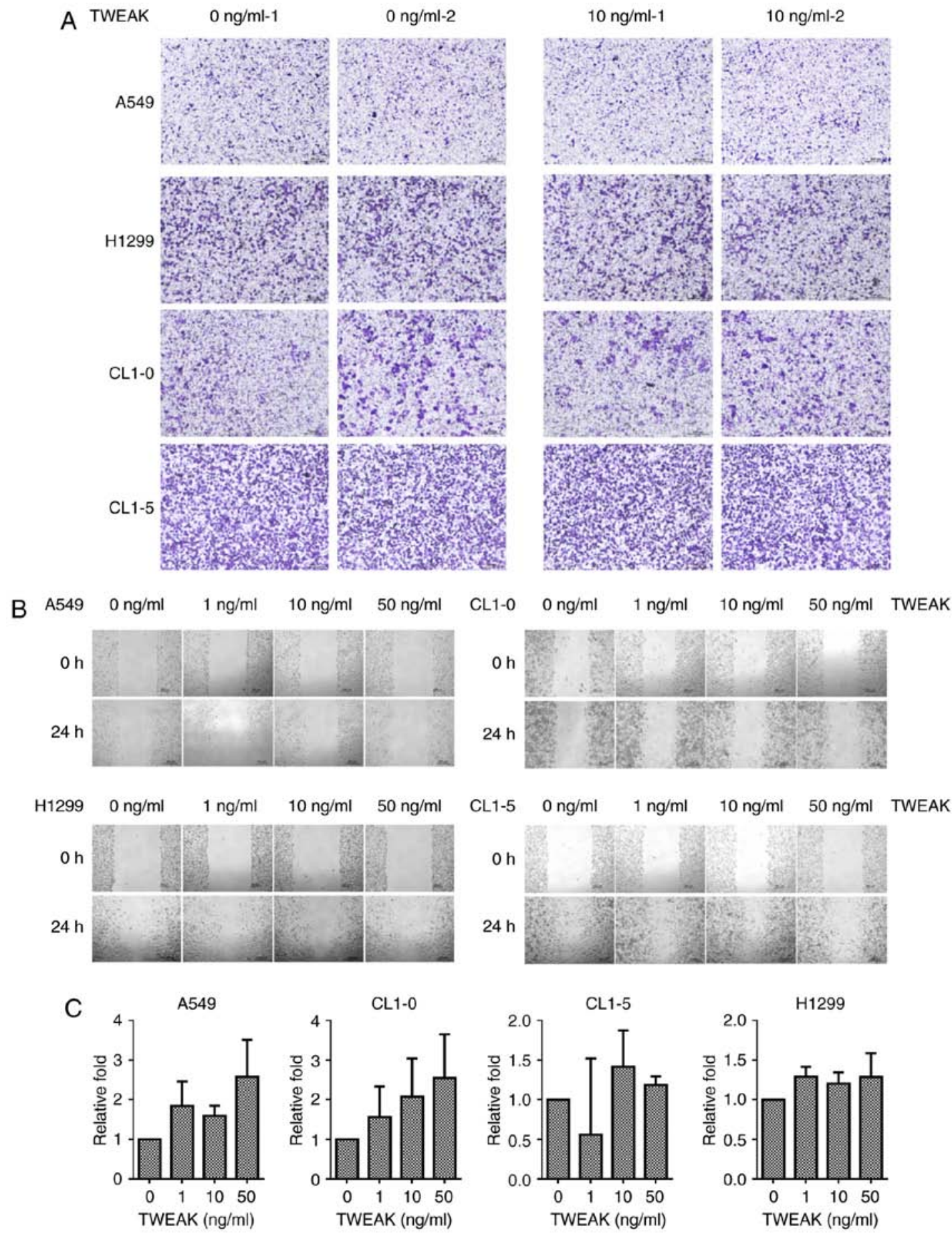

Figure 4. Effect of TWEAK treatment on cell migration. Evaluating dose effect of TWEAK on (A) Transwell and (B) wound healing assays, and (C) quantification of wound healing assay. The error bars represent SD.

(GSE7670) (31,32). Compared to the SCLC cell line H209 which was observed to have low TWEAK expression and the NSCLC cell line Calu-3 which is reported to express a high level of Fn14 (22), Fn14 expression in A549 was similar to that in Calu-3 and higher than that in H209 (Fig. 3A). As shown in Fig. 3B, differential Fn14 expression among the four cell lines was observed through analysis of GSE7670. The highest level of TWEAK and Fn14 was observed in A549 cells. Our results showed that the proliferation rate of lung cancer cell lines was not enhanced and inhibited by the treatment of different doses of TWEAK in all cell lines (Fig. 3C-F). In addition, the results of Transwell and wound-healing assays revealed that TWEAK did not affect the migration capacity (Fig. 4A-C). Even though TWEAK expression was different, TWEAK treatment did not promote cell proliferation and migration among the four cell lines.

Detection of serum TWEAK concentration in patients with lung cancer. Bioinformatic and in vitro analysis showed that TWEAK expression in NSCLC and exogenous TWEAK treatment did not promote tumor progression. To further investigate the association between TWEAK and lung cancer, 
A

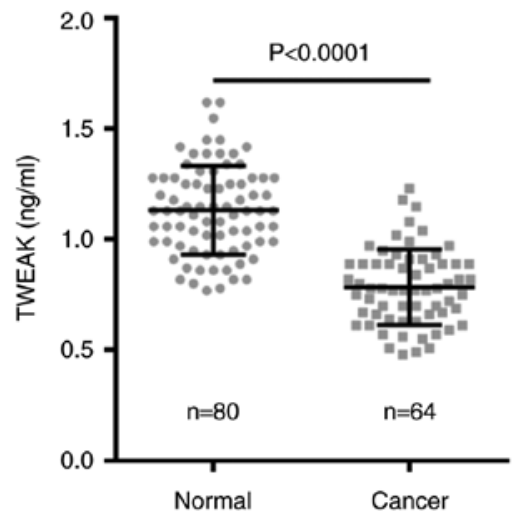

C

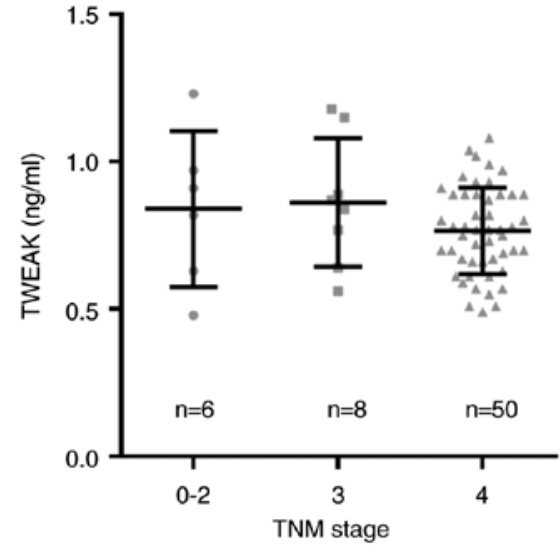

B

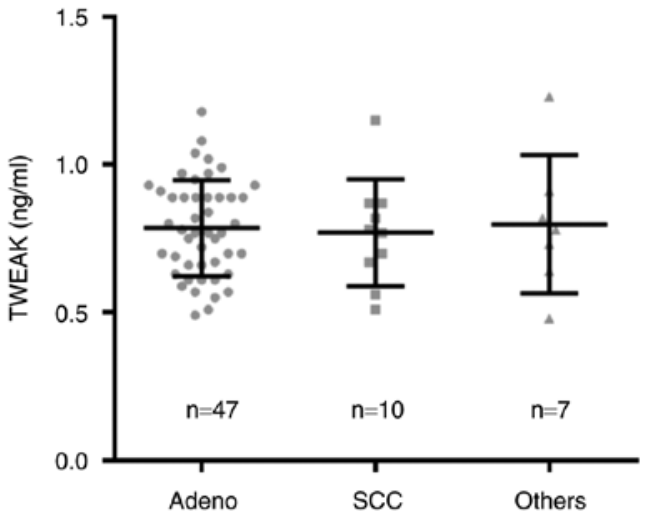

D

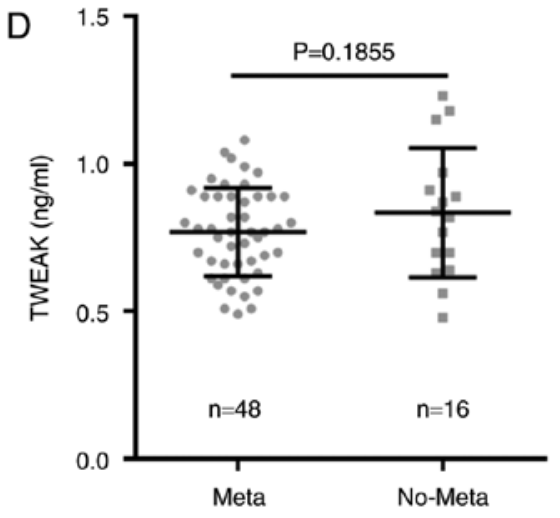

Figure 5. TWEAK levels in serum of patients with non-small cell lung cancer. (A) TWEAK levels in healthy donors and patients. (B) TWEAK levels in patients with different types of non-small cell lung cancer including adenocarcinoma (Adeno), squamous cell carcinoma (SCC), and other types (other). (C) TWEAK levels in patients with different TNM stage. (D) TWEAK levels in patients with metastatic (Meta) and non-metastatic (Non) tumors.

Table II. Characteristics of healthy controls and lung cancer patients.

\begin{tabular}{lcc}
\hline & $\begin{array}{c}\text { Healthy control } \\
(\mathrm{n}=80)\end{array}$ & $\begin{array}{c}\text { Lung cancer } \\
(\mathrm{n}=64)\end{array}$ \\
\hline Age (years) & $62.95 \pm 10.14$ & $65.88 \pm 10.79$ \\
Sex (male/female) & $51 / 29$ & $33 / 31$ \\
Lung cancer & & \\
Adenocarcinoma & & 47 \\
Squamous cell carcinoma & & 10 \\
Other types & & 7 \\
TNM stage & & \\
$0-2$ & & 6 \\
3A+3B & & 50 \\
4 & & \\
Metastasis & & 48 \\
Yes & & 16 \\
No & & \\
\hline
\end{tabular}

TNM, tumour, node and metastasis.

the sera of 64 NSCLC patients which included 47 patients with adenocarcinoma, 10 patients with squamous cell carcinoma, and 7 patients with other types of NSCLC, and 80 normal controls were collected. The characteristics of the patients and normal controls are listed in Table II. Our results showed that TWEAK levels in normal controls were significantly higher than that in NSCLC patients (Fig. 5A). This may imply that TWEAK plays a different role in healthy individuals and NSCLC patients. In addition, NSCLC patients were grouped by their cell type (Fig. 5B), TNM stage (Fig. 5C), and metastasis status (Fig. 5D). There were no significantly different levels in TWEAK among these groups.

\section{Discussion}

In multiple types of human cancer, TWEAK/Fn14 signaling pathways promote cell growth, metastasis and invasion in vitro and in vivo. It suggests that TWEAK/Fn14 could serve as a potential therapeutic target. Anti-TWEAK mAb RG7212 exerts an antitumor effect in various types of cancer cell lines, including the NSCLC cell line Calu-3 which expresses a high level of Fn14 (22). In addition, the therapeutic effect of RG7212 is associated with Fn14 expression (22). A study of a phase I clinical trial of RG7212 which is a TWEAK antibody showed that RG7272 treatment is safe (36). In patients with solid tumors (including two NSCLC patients), only high exposure of RG7212 treatment resulted in a decrease in the Fn14 signaling pathway (37). This indicates that targeting TWEAK/Fn14 signaling pathway may be a novel antitumor strategy. Our bioinformatic analysis indicated that Fn14 expression in adenocarcinoma is higher than that in normal 


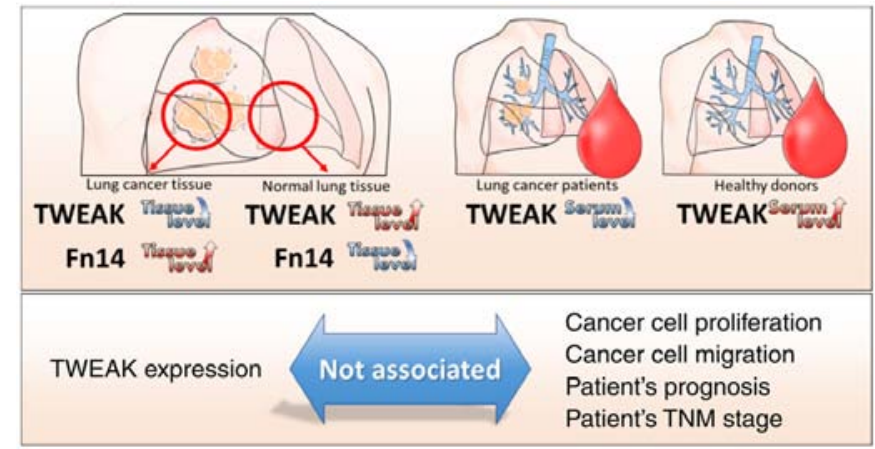

Figure 6. Proposed model scheme of TWEAK in non-small cell lung cancer.

tissue. This may imply that RG7212 is beneficial for treating lung adenocarcinoma.

In contrast, bioinformatic analysis showed lower TWEAK expression in both lung adenocarcinoma and squamous cell carcinoma. Therefore, we suppose that TWEAK which is secreted from tumor-infiltrating immune cells may promote tumor progression in physiological condition. Surprisingly, TWEAK treatment did not trigger cell proliferation and migration in four NSCLC cell lines even though endogenous TWEAK and Fn14 expression was found to be different among these cell lines. Furthermore, the serum TWEAK level was significantly low in patient compared to normal tissues. This may imply that endogenous TWEAK expression and TWEAK treatment may not be involved in Fn14 signaling pathway-mediated NSCLC progression.

Unique TWEAK/Fn14 expression and serum TWEAK concentrations were observed in the present study, in ovarian cancer patients and head and neck cancer patients. TWEAK expression was detected in malignant ovarian cancer tissues but the expression of TWEAK/Fn14 did not correlate with the patient subtype, stage, or pathological features (38). In the patients with head and neck cancer, a low serum TWEAK level was found to be associated with a poor recurrence-free survival rate (39). CD163 which is another receptor of TWEAK may be a potential factor which affects TWEAK/Fn14 signaling pathways (40). CD163 is expressed on the surface on monocytes and macrophages and the soluble form of CD163 is constitutively detected in serum (41). Bover et al proposed that CD163 may serve as a scavenger of soluble TWEAK in pathological condition (42). Thus, we hypothesized that the interaction of TWEAK and Fn14 may be a critical factor affecting the TWEAK/Fn14 signaling pathway in the tumor microenvironment in lung cancer, particularly in NSCLC. Investigation of soluble CD163 level and CD163/TWEAK interaction in tumor-infiltrating macrophages may provide a novel regulatory mechanism. In addition, the effect of anti-TWEAK antibody RG7212 on CD163/TWEAK may warrant further investigation in animal tumor models or clinical trials in NSCLC or lung adenocarcinoma.

Compared to normal lung tissues, higher level of Fn14 was observed in the bioinformatic analysis (Fig. 1A). High Fn14 expression was associated with poor survival in lung adenocarcinoma (Fig. 2D). This finding suggests that the Fn14 signaling pathway plays a critical role in tumor progression. Previous studies have demonstrated that Fn14 expression is enhanced by Src, HGFR/MET, and EGFR mutation (exon 19 deletion) (24-26). These factors may also affect CD163 and TWEAK interaction and downstream signaling pathways. Therefore, the role of TWEAK should be investigated in consideration of CD163 and Fn14 expression and the status of other signaling molecules in the clinic.

In NSCLC, our results suggest that TWEAK expression is relatively low and Fn14 expression is high compared to normal lung tissues. In addition, TWEAK expression is not associated with a relapse-free survival rate but high Fn14 expression is associated with poor prognosis in lung adenocarcinoma. We found that TWEAK treatment did not promote cell proliferation and migration. Although the TWEAK-mediated signaling pathways could not be evaluated in serum samples, and the serum sample size was small in the present study, our results revealed that a low serum TWEAK level may be a feature of NSCLC in the clinic (Fig. 6). Further studies on the interaction among TWEAK, soluble CD136, and Fn14 may further clarify the role of TWEAK in NSCLC.

\section{Acknowledgements}

The present study was supported by grants from the Ministry of Science and Technology (MOST 106-2314-B037-016-MY2; MOST 104-2314-B-037-053-MY4; MOST 104-2320-B-037-014-MY3; MOST 103-2320-B-037-006MY3), the KMU-KMUH Co-Project of Key Research (grant no. KMU-DK 107009 from Kaohsiung Medical University) and the Kaohsiung Medical University Hospital Research Foundation (KMUHS10601; KMUH105-5R14).

\section{References}

1. Siegel RL, Miller KD and Jemal A: Cancer Statistics, 2017. CA Cancer J Clin 67: 7-30, 2017.

2. Mar N, Vredenburgh JJ and Wasser JS: Targeting HER2 in the treatment of non-small cell lung cancer. Lung Cancer 87: 220-225, 2015.

3. Kazandjian D, Blumenthal GM, Chen HY, He K, Patel M, Justice R, Keegan P and Pazdur R: FDA approval summary: Crizotinib for the treatment of metastatic non-small cell lung cancer with anaplastic lymphoma kinase rearrangements. Oncologist 19: e5-e11, 2014.

4. Kris MG, Johnson BE, Berry LD, Kwiatkowski DJ, Iafrate AJ, Wistuba II, Varella-Garcia M, Franklin WA, Aronson SL, Su PF, et al: Using multiplexed assays of oncogenic drivers in lung cancers to select targeted drugs. JAMA 311: 1998-2006, 2014.

5. Rolfo C, Caglevic C, Santarpia M, Araujo A, Giovannetti E, Gallardo CD, Pauwels P and Mahave M: Immunotherapy in NSCLC: A Promising and revolutionary Weapon. Adv Exp Med Biol 995: 97-125, 2017.

6. Multhaupt HA, Leitinger B, Gullberg D and Couchman JR: Extracellular matrix component signaling in cancer. Adv Drug Deliv Rev 97: 28-40, 2016.

7. El-Nikhely N, Larzabal L, Seeger W, Calvo A and Savai R: Tumor-stromal interactions in lung cancer: Novel candidate targets for therapeutic intervention. Expert Opin Investig Drugs 21: 1107-1122, 2012

8. Kataki A, Scheid P, Piet M, Marie B, Martinet N, Martinet Y and Vignaud JM: Tumor infiltrating lymphocytes and macrophages have a potential dual role in lung cancer by supporting both host-defense and tumor progression. J Lab Clin Med 140: 320-328, 2002.

9. Carus A, Ladekarl M, Hager H, Pilegaard H, Nielsen PS and Donskov F: Tumor-associated neutrophils and macrophages in non-small cell lung cancer: No immediate impact on patient outcome. Lung Cancer 81: 130-137, 2013. 
10. Hsu YL, Hung JY, Ko YC, Hung CH, Huang MS and Kuo PL: Phospholipase D signaling pathway is involved in lung cancer-derived IL-8 increased osteoclastogenesis. Carcinogenesis 31: 587-596, 2010

11. Kuo PL, Huang MS, Hung JY, Chou SH, Chiang SY, Huang YF, Yang CJ, Tsai MJ, Chang WA and Hsu YL: Synergistic effect of lung tumor-associated dendritic cell-derived HB-EGF and CXCL5 on cancer progression. Int J Cancer 135: 96-108, 2014.

12. Rivas-Fuentes S, Salgado-Aguayo A, Pertuz Belloso S, Gorocica Rosete P, Alvarado-Vásquez N and Aquino-Jarquin G: Role of chemokines in non-small cell lung cancer: Angiogenesis and inflammation. J Cancer 6: 938-952, 2015.

13. van Horssen R, Ten Hagen TL and Eggermont AM: TNF-alpha in cancer treatment: Molecular insights, antitumor effects, and clinical utility. Oncologist 11: 397-408, 2006.

14. Lebrec H, Ponce R, Preston BD, Iles J, Born TL and Hooper M: Tumor necrosis factor, tumor necrosis factor inhibition, and cancer risk. Curr Med Res Opin 31: 557-574, 2015.

15. Winkles JA: The TWEAK-Fn14 cytokine-receptor axis: Discovery, biology and therapeutic targeting. Nat Rev Drug Discov 7: 411-425, 2008.

16. Willis AL, Tran NL, Chatigny JM, Charlton N, Vu H, Brown SA, Black MA, McDonough WS, Fortin SP, Niska JR, et al: The fibroblast growth factor-inducible 14 receptor is highly expressed in HER2-positive breast tumors and regulates breast cancer cell invasive capacity. Mol Cancer Res 6: 725-734, 2008.

17. Huang M, Narita S, Tsuchiya N, Ma Z, Numakura K, Obara T, Tsuruta H, Saito M, Inoue T, Horikawa Y, et al: Overexpression of Fn14 promotes androgen-independent prostate cancer progression through MMP-9 and correlates with poor treatment outcome. Carcinogenesis 32: 1589-1596, 2011.

18. Kwon OH, Park SJ, Kang TW, Kim M, Kim JH, Noh SM, Song KS, Yoo HS, Wang Y, Pocalyko D, et al: Elevated fibroblast growth factor-inducible 14 expression promotes gastric cancer growth via nuclear factor-kB and is associated with poor patient outcome. Cancer Lett 314: 73-81, 2012.

19. Tran NL, McDonough WS, Savitch BA, Fortin SP, Winkles JA, Symons M, Nakada M, Cunliffe HE, Hostetter G, Hoelzinger DB, et al: Increased fibroblast growth factor-inducible 14 expression levels promote glioma cell invasion via Rac1 and nuclear factor-kappaB and correlate with poor patient outcome. Cancer Res 66: 9535-9542, 2006.

20. Cherry EM, Lee DW, Jung JU and Sitcheran R: Tumor necrosis factor-like weak inducer of apoptosis (TWEAK) promotes glioma cell invasion through induction of $N F-\kappa B$-inducing kinase (NIK) and noncanonical NF- $\kappa \mathrm{B}$ signaling. Mol Cancer 14: 9 , 2015.

21. Armstrong CL, Galisteo R, Brown SA and Winkles JA: TWEAK activation of the non-canonical NF- $\mathrm{BB}$ signaling pathway differentially regulates melanoma and prostate cancer cel invasion. Oncotarget 7: 81474-81492, 2016.

22. Yin XF, Luistro L, Zhong H, Smith M, Nevins T, Schostack K, Hilton H, Lin TA, Truitt T, Biondi D, et al: RG7212 Anti-TWEAK mAb inhibits tumor growth through Inhibition of tumor cell proliferation and survival signaling and by enhancing the host antitumor immune response. Clin Cancer Res 19: 5686-5698, 2013.

23. Zou H, Wang D, Gan X, Jiang L, Chen C, Hu L and Zhang Y: Low TWEAK expression is correlated to the progression of squamous cervical carcinoma. Gynecol Oncol 123: 123-128, 2011.

24. Cheng E, Whitsett TG, Tran NL and Winkles JA: The TWEAK receptor Fn14 is an Src-inducible protein and a positive regulator of Src-driven cell invasion. Mol Cancer Res 13 $575-583,2015$

25. Sun Y, Han Y, Wang X, Wang W, Wang X, Wen M, Xia J, Xing H, Li X and Zhang Z: Correlation of EGFR Del 19 with Fn14/JAK/STAT signaling molecules in non-small cell lung cancer. Oncol Rep 36: 1030-1040, 2016.

26. Whitsett TG, Fortin Ensign SP, Dhruv HD, Inge LJ, Kurywchak P, Wolf KK, LoBello J, Kingsley CB, Allen JW, Weiss GJ and Tran NL: FN14 expression correlates with MET in NSCLC and promotes MET-driven cell invasion. Clin Exp Metastasis 31: $613-623,2014$
27. Bhattacharjee A, Richards WG, Staunton J, Li C, Monti S, Vasa P, Ladd C, Beheshti J, Bueno R, Gillette M, et al: Classification of human lung carcinomas by mRNA expression profiling reveals distinct adenocarcinoma subclasses. Proc Natl Acad Sci USA 98 13790-13795, 2001.

28. Hou J, Aerts J, den Hamer B, van Ijcken W, den Bakker M, Riegman P, van der Leest C, van der Spek P, Foekens JA, Hoogsteden HC, et al: Gene expression-based classification of non-small cell lung carcinomas and survival prediction. PLoS One 5: e10312, 2010

29. Landi MT, Dracheva T, Rotunno M, Figueroa JD, Liu H, Dasgupta A, Mann FE, Fukuoka J, Hames M, Bergen AW, et al: Gene expression signature of cigarette smoking and its role in lung adenocarcinoma development and survival. PLoS One 3 : e1651, 2008.

30. Selamat SA, Chung BS, Girard L, Zhang W, Zhang Y, Campan M, Siegmund KD, Koss MN, Hagen JA, Lam WL, et al: Genome-scale analysis of DNA methylation in lung adenocarcinoma and integration with mRNA expression. Genome Res 22: 1197-1211, 2012.

31. Su LJ, Chang CW, Wu YC, Chen KC, Lin CJ, Liang SC, Lin CH, Whang-Peng J, Hsu SL, Chen CH, et al: Selection of DDX5 as a novel internal control for Q-RT-PCR from microarray data using a block bootstrap re-sampling scheme. BMC Genomics 8: 140 , 2007.

32. Barretina J, Caponigro G, Stransky N, Venkatesan K, Margolin AA, Kim S, Wilson CJ, Lehár J, Kryukov GV, Sonkin D, et al: The cancer cell line encyclopedia enables predictive modelling of anticancer drug sensitivity. Nature 483: 603-607, 2012.

33. Győrffy B, Surowiak P, Budczies J and Lanczky A: Online survival analysis software to assess the prognostic value of biomarkers using transcriptomic data in non-small-cell lung cancer. PLoS One 8: e82241, 2013.

34. Chu YW, Yang PC, Yang SC, Shyu YC, Hendrix MJ, Wu R and Wu CW: Selection of invasive and metastatic subpopulations from a human lung adenocarcinoma cell line. Am J Respir Cell Mol Biol 17: 353-360, 1997.

35. WHO: World cancer report 2014. In: Chapter 5.1. 2014.

36. Lassen UN, Meulendijks D, Siu LL, Karanikas V, Mau-Sorensen M, Schellens JH, Jonker DJ, Hansen AR, Simcox ME, Schostack KJ, et al: A phase I monotherapy study of RG7212, a first-in-class monoclonal antibody targeting TWEAK signaling in patients with advanced cancers. Clin Cancer Res 22: 258-266, 2015.

37. Meulendijks D, Lassen UN, Siu LL, Huitema AD, Karanikas V, Mau-Sorensen M, Jonker DJ, Hansen AR, Simcox ME, Schostack KJ, et al: Exposure and tumor Fn14 expression as determinants of pharmacodynamics of the anti-TWEAK monoclonal antibody RG7212 in patients with Fn14-positive solid tumors. Clin Cancer Res 22: 858-867, 2016.

38. GuL,DaiL,CaoC,ZhuJ,Ding C,XuHB,QiuLandDiW:Functional expression of TWEAK and the receptor Fn14 in human malignant ovarian tumors: Possible implication for ovarian tumor intervention. PLoS One 8: e57436, 2013.

39. Aviles-Jurado FX, Terra X, Gomez D, Flores JC, Raventós A, Maymó-Masip E, León X, Serrano-Gonzalvo V, Vendrell J, Figuerola E and Chacón MR: Low blood levels of sTWEAK are related to locoregional failure in head and neck cancer. Eur Arch Otorhinolaryngol 272: 1733-1741, 2015.

40. Moreno JA, Muñoz-Garcia B, Martin-Ventura JL, Madrigal-Matute J, Orbe J, Páramo JA, Ortega L, Egido J and Blanco-Colio LM: The CD163-expressing macrophages recognize and internalize TWEAK: Potential consequences in atherosclerosis. Atherosclerosis 207: 103-110, 2009.

41. Tang X: Tumor-associated macrophages as potential diagnostic and prognostic biomarkers in breast cancer. Cancer Lett 332: 3-10,2013.

42. Bover LC, Cardó-Vila M, Kuniyasu A, Sun J, Rangel R, Takeya M, Aggarwal BB, Arap W and Pasqualini R: A previously unrecognized protein-protein interaction between TWEAK and CD163: Potential biological implications. J Immunol 178: 8183-8194, 2007. 